\title{
Emerging Imaging Modalities in Regenerative Medicine
}

\author{
Mitchel R. Stacy • Albert J. Sinusas
}

Published online: 28 January 2015

(c) Springer Science+Business Media New York 2015

\begin{abstract}
The field of regenerative medicine has experienced considerable growth in recent years as the translation of pre-clinical biomaterials and cell- and gene-based therapies begin to reach clinical application. Until recently, the ability to monitor the serial responses to therapeutic treatments has been limited to post-mortem tissue analyses. With improvements in existing imaging modalities and the emergence of hybrid imaging systems, it is now possible to combine information related to structural remodeling with associated molecular events using non-invasive imaging. This review summarizes the established and emerging imaging modalities that are available for in vivo monitoring of clinical regenerative medicine therapies and discusses the strengths and limitations of each imaging modality.
\end{abstract}

Keywords Regenerative medicine - Molecular imaging · Cell therapy $\cdot$ Gene therapy $\cdot$ Biomaterials $\cdot$ Tissue engineering

This article is part of the Topical Collection on Tissue Engineering and Regeneration.

M. R. Stacy $(\bowtie)$

Department of Internal Medicine, Yale University

School of Medicine, P.O. Box 208017, Dana-3, New Haven, CT 06520, USA

e-mail: mitchel.stacy@yale.edu

\section{A. J. Sinusas}

Departments of Internal Medicine \& Diagnostic Radiology,

Yale University School of Medicine, P.O. Box 208017, Dana-3,

New Haven, CT 06520, USA

e-mail: albert.sinusas@yale.edu

\section{Introduction}

Regenerative medicine aims to replace, regenerate, or restore function within damaged or diseased organs, tissue, and cells through a variety of technologies and approaches such as gene therapy, stem and progenitor cell therapy, and tissue engineering [1]. Many physiological systems have served as targets for regenerative therapies; however, evaluation of the effectiveness of regenerative approaches has traditionally been limited by the inability to serially monitor implanted cells or biomaterials and physiological responses to therapy. Specifically, the fate of gene- and cell-based therapies, as well as the rate of scaffold degradation and timing of associated molecular events, has been challenging to track in vivo [2].

Recent advancements in functional and targeted imaging agents as well as clinical imaging systems are beginning to offer novel techniques for the assessment of serial responses to various forms of regenerative therapies. Primary clinical imaging tools consist of ultrasound, X-ray computed tomography (CT), single photon emission tomography (SPECT), positron emission tomography (PET), and magnetic resonance (MR) imaging. Although each modality has advantages and disadvantages (Table 1), the emergence of hybrid imaging systems such as SPECT/CT, PET/CT, and PET/MR now allows for the co-registration of high-sensitivity radiotracerbased molecular imaging with higher-resolution anatomical CT or MR imaging. These multimodality systems enable application of anatomic information from CT or MR for colocalization and absolute quantification of radiotracers with correction of scatter and attenuation and partial volume errors that complicate radiotracer-based imaging, decreasing imaging artifacts from surrounding non-target soft tissues. More specifically, the result of combining structural and functional imaging modalities has improved quantification of uptake for 
Table 1 Benefits and limitations of clinical imaging modalities

\begin{tabular}{|c|c|c|c|c|c|}
\hline Modality & Sensitivity & $\begin{array}{l}\text { Penetration } \\
\text { depth }\end{array}$ & $\begin{array}{l}\text { Spatial } \\
\text { resolution }\end{array}$ & Advantages & Disadvantages \\
\hline Ultrasound & Moderate & Low & $1 \mathrm{~mm}$ & $\begin{array}{l}\text { Widely available; inexpensive; no } \\
\text { ionizing radiation; real-time imaging }\end{array}$ & $\begin{array}{l}\text { Limited molecular probes; small field of view; } \\
\text { operator dependent }\end{array}$ \\
\hline MR & Moderate & No limit & $<1-3 \mathrm{~mm}^{3}$ & $\begin{array}{l}\text { No ionizing radiation; high spatial } \\
\text { resolution }\end{array}$ & $\begin{array}{l}\text { Susceptibility to motion artifacts; limited } \\
\text { molecular probes; not compatible for } \\
\text { patients with metallic implants or renal } \\
\text { insufficiency; long imaging times }\end{array}$ \\
\hline SPECT & High & No limit & $\sim 5-8 \mathrm{~mm}^{3}$ & $\begin{array}{l}\text { High sensitivity; multiple radiotracers } \\
\text { available for molecular imaging; } \\
\text { ability to serially monitor long half- } \\
\text { life isotopes }\end{array}$ & $\begin{array}{l}\text { Exposure to ionizing radiation; attenuation } \\
\text { from low-energy photons }\end{array}$ \\
\hline PET & High & No limit & $\sim 3-5 \mathrm{~mm}^{3}$ & $\begin{array}{l}\text { High sensitivity; established methods } \\
\text { for attenuation correction; accurate } \\
\text { and precise quantification }\end{array}$ & $\begin{array}{l}\text { Exposure to ionizing radiation; need for on- } \\
\text { site cyclotron or generator; advanced } \\
\text { radiochemistry }\end{array}$ \\
\hline $\mathrm{CT}$ & Limited & No limit & $<1 \mathrm{~mm}^{3}$ & $\begin{array}{l}\text { High spatial resolution can be combined } \\
\text { with other modalities (PET/SPECT) }\end{array}$ & $\begin{array}{l}\text { Exposure to ionizing radiation; patient } \\
\text { sensitivity to iodinated contrast agents }\end{array}$ \\
\hline OCT & High & Low & $10-20 \mu \mathrm{m}$ & $\begin{array}{l}\text { High-resolution intravascular imaging; } \\
\text { superior plaque imaging }\end{array}$ & $\begin{array}{l}\text { Catheterization required; poor penetration } \\
\text { depth }\end{array}$ \\
\hline
\end{tabular}

Modified from Naumova et al. [2]

targeted molecular radiotracers with improved localization to specific sites of regenerative therapies [3]. Molecular imaging has been used to evaluate physiological and pathological processes in response to regenerative therapies, with common clinical applications focused on various forms of cancer [4], cardiovascular [5, 6] and metabolic [7••, 8, 9] disease, and neurological trauma [10,11].

In addition to established tools for non-invasive assessment of physiological processes, recent imaging work has focused on optimizing molecular probes that can allow for in vivo serial tracking of transplanted cells for clinical regenerative medicine. Current technology for tracking transplanted cells in humans first requires cell labeling via direct or indirect labeling. Direct labeling consists of using molecular probes (nanoparticles or radiotracers) that are actively/passively transported and trapped within cells, whereas indirect labeling involves transfection of cells using reporter gene constructs that are integrated into the cellular genome by viral or non-viral vectors. Both labeling strategies are capable of producing an imaging signal that is detectable by modalities such as SPECT, PET, and MR [12]. Reporter gene labeling appears to be the ideal labeling technique for non-invasive tracking of transplanted cell fate for a period of weeks, months, or years; however, clinical trials to date have primarily utilized direct labeling techniques to track cells, which may be due to ongoing safety concerns related to genomic manipulations and prolonged behavior of gene products within patients [13]. Ultimately, the success of clinical regenerative trials will be dependent on the engraftment, survival, and integration of transplanted cells into targeted tissue or organs. Ongoing problems remain with regard to optimizing the site and timing of cell delivery, and clinical trials utilizing non-invasive imaging for tracking of cell fate have to date demonstrated poor rates of cell retention, irrespective of the methodology incorporated for cell delivery. Further development and application of molecular imaging in clinical trials should improve evaluation of cell fate in target tissue and lead to quantitative tools for non-invasive tracking of transplanted cells [12].

The following sections review the most established and emerging clinical imaging modalities that have been used to target gene- and cell-based therapies and describe their past, present, and potential applications in regenerative medicine clinical trials. Additionally, relative advantages, limitations, and potential safety issues are discussed for each imaging approach.

\section{Radiotracer Imaging}

SPECT and PET are the primary nuclear imaging modalities that can be used for radiotracer-based evaluation of a variety of molecular processes, such as angiogenesis, inflammation, and metabolism [14]. Both SPECT and PET provide three-dimensional functional images through the detection of gamma rays that are emitted from isotopes having varying half-lives and energies and have been effectively used for targeted imaging. Although SPECT and PET provide the highest sensitivity out of the whole body clinical modalities for molecular imaging, both 
possess low spatial resolution, and the use of isotopes exposes patients to ionizing radiation. Due to the limited spatial resolution of nuclear modalities, high-sensitivity SPECT and PET images are commonly paired with CT or MR images for co-localization with high-resolution anatomical information. To date, the inability of SPECT and PET to evaluate constructs at high resolution has limited their application in tissue-engineering clinical trials; however, recent pre-clinical work has demonstrated the potential of hybrid SPECT/CT imaging for detecting scaffold remodeling in tissue-engineered vascular grafts through targeted imaging of matrix metalloproteinase activity [15].

Nuclear approaches have been the most frequently utilized modalities in evaluating the biodistribution of transplanted cells due to their high sensitivity for non-invasive detection of radiolabeled cells $\left(\sim 10^{4}-10^{6}\right.$ cells/voxel $)$ [12]. However, one main limitation of applying SPECT and PET for regenerative trials is the short half-lives of standard isotopes used for clinical imaging, which currently limits the ability to perform long-term tracking of radiolabeled cell fate. Direct labeling of cells has primarily been applied in clinical trials using common SPECT isotopes such as technetium-99 $\mathrm{m}\left({ }^{99 \mathrm{~m}} \mathrm{Tc} ; 6 \mathrm{~h}\right.$ half-life $)$ or indium-111 ( ${ }^{111} \mathrm{In}$; $67 \mathrm{~h}$ half-life $)$, and the PET isotope Fluorine-18 $\left({ }^{18} \mathrm{~F}\right.$; $\sim 110$ min half-life)-labeled deoxyglucose, with the majority of these SPECT and PET studies focused on tracking effective delivery to and localization of radiolabeled cells in the myocardium of patients following myocardial infarction [5, 6, 16, 17, 18••]. Problems that still exist when applying cell therapy in patients with myocardial infarction are selection and optimization of cell delivery technique (i.e., intracoronary or intramyocardial injection), the ability to non-invasively evaluate retention of transplanted cells, and the death and ultimate phagocytosis of transplanted cells [2]. To date, clinical trials using various cell labeling strategies for SPECT and PET imaging have demonstrated relatively low cell retention in the myocardium of patients (ranging from $\sim 1$ to $25 \%$ of injected cell dose), regardless of delivery technique [12]. However, Vrtovec et al. [18••] found higher cell retention at $18 \mathrm{~h}$ following cell transplantation using transendocardial compared to intracoronary delivery of CD34+ cells labeled with ${ }^{99 \mathrm{~m}} \mathrm{Tc}$, which was also associated with greater improvements in left ventricular function at 6 months following treatment. To date, there is not one specific delivery technique or direct cell labeling strategy that has proven to be superior in clinical trials, and the ability to perform long-term tracking of transplanted cells with SPECT and PET remains elusive.

A potential solution to long-term cell tracking may exist in the development of novel reporter genes, which allow for SPECT or PET imaging of viable transfected cells that retain radiotracers following intravenous injection and can thus be imaged at various time points after initial cell transplant. The first clinical trial to incorporate reporter gene technology was performed in a patient with glioblastoma and used the PET reporter ${ }^{18} \mathrm{~F}$-9-[4-fluoro-3(hydroxymethyl)butyl]guanine $\left({ }^{18} \mathrm{~F}-\mathrm{FHBG}\right)$ to non-invasively image genetically modified cytolytic CD8 $+\mathrm{T}$ cells that expressed the interleukin 13 zetakine gene and herpes simplex virus thymidine kinase type 1 (HSV1-tk). Expression of interleukin 13 zetakine allowed for transplanted $\mathrm{T}$ cells to target receptor proteins of tumor cells while HSV1-tk phosphorylated ${ }^{18} \mathrm{~F}-\mathrm{FHBG}$, therefore permitting non-invasive assessment of localized radiotracer uptake in tumor cells within the brain [19]. Another genebased approach that has been applied in pre-clinical models that have potential for clinical translation is the sodium iodide symporter (NIS) [20, 21 ••]. Viable transplanted cells transfected with NIS can be detected non-invasively using SPECT imaging of iodide-123 $\left({ }^{123} \mathrm{I}, 13 \mathrm{~h}\right.$ half-life) or ${ }^{99 \mathrm{~m}} \mathrm{Tc}$, as both isotopes are taken up by NIS and retained following intravenous or intra-arterial administration. Pre-clinical application of NIS in a porcine model of myocardial infarction has already demonstrated the ability to visualize viable cells for up to 15 weeks following myocardial transplantation [21••]. Additionally, SPECT ${ }^{99 \mathrm{~m}}$ Tc-tetrofosmin imaging and NOGA electromechanical mapping have been combined with SPECT imaging of ${ }^{123}$ I-labeled NIS-transfected cells to guide delivery and evaluate localization of cell transplantation in infarct border zones of the porcine heart (Fig. 1) [22]. Future clinical translation of NIS-based approaches is encouraging, as NIS is a human protein that should not stimulate an immunogenic response. Furthermore, this approach could be extended to PET imaging of NIS in the pre-clinical or clinical setting with the use of PET isotopes ${ }^{124} \mathrm{I}$ (4.2 day half-life) and ${ }^{94 \mathrm{~m}} \mathrm{Tc}$ (52.5 min half-life), respectively.

In addition to tracking of transplanted cells in the setting of myocardial infarction, nuclear imaging approaches have also been applied to evaluate early cell retention in the human brain [23••, 24] and liver [8, 9]. Specifically, scintigraphy of ${ }^{99 \mathrm{~m}} \mathrm{Tc}$-labeled bone marrow mononuclear cells has allowed for imaging of cell retention in the brain of stroke patients at 2 and $24 \mathrm{~h}$ following injection and demonstrated similar levels of cell retention using intraarterial or intravenous cell delivery [23••]. PET imaging of ${ }^{18} \mathrm{~F}$-DOPA has been used to evaluate maturation of fetal neurons in patients with Parkinson's disease, and the PET agent raclopride, which is a dopamine D2 receptor-binding agent, has been used to quantitatively evaluate neurochemical tone in Parkinson's patients [25, 26]. Additionally, PET imaging of raclopride has proven to be an effective technique for evaluating endogenous dopamine in cell transplants up to 15 years post-transplantation [27••]. 


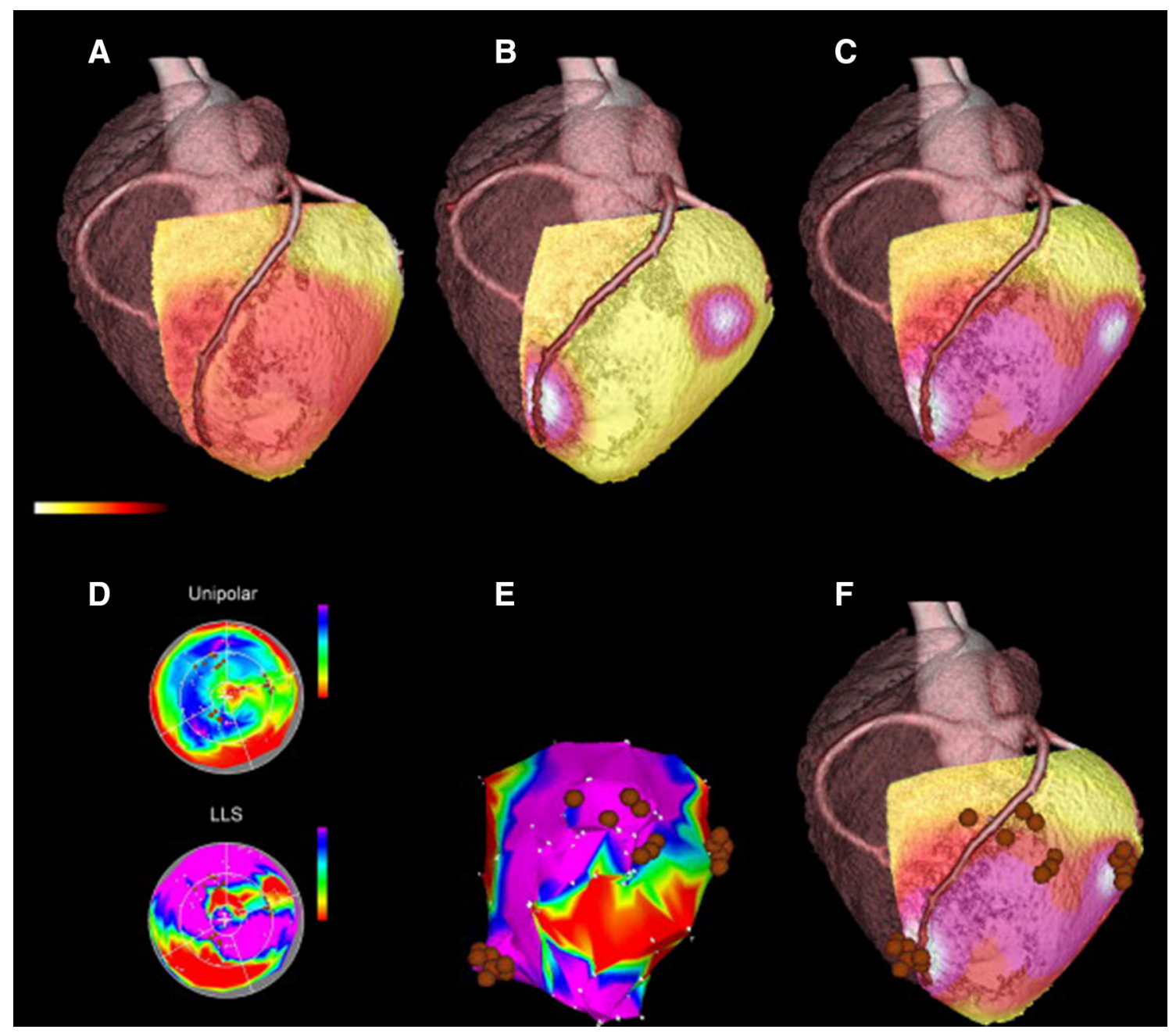

Fig. 1 Fusion of in vivo multimodality imaging in a porcine model of myocardial infarction following intramyocardial transplantation of human-induced pluripotent stem cells (hiPSCs) transfected with NIS. a SPECT imaging of ${ }^{99 \mathrm{~m}} \mathrm{Tc}$-tetrofosmin fused with coronary CT angiography (CTA) allowed for visualization of perfusion defect and coronary anatomy in the anterior wall of the left ventricle. b Fusion of coronary CTA and SPECT imaging of NIS-transfected cells labeled with ${ }^{123} \mathrm{I}$ demonstrated focal ${ }^{123} \mathrm{I}$ hot spots localized to the sites of cell injection. c Fusion of CTA, SPECT ${ }^{99 \mathrm{~m}}$ Tc-tetrofosmin, and SPECT ${ }^{123}$ I permitted visualization of perfusion defect (identified as red/pink) and NIS-transfected cells (identified as white areas) located near the

Application of PET imaging for evaluating ${ }^{18}$ F-FDGlabeled islets transplanted in the liver of diabetic patients has also demonstrated the safety and potential of this approach for evaluation of cell engraftment $[8,9]$.

Ongoing development of SPECT and PET imaging approaches for long-term tracking of labeled cells should greatly enhance the application of nuclear modalities in regenerative clinical trials. In addition to tracking of cell fate, nuclear approaches may also permit evaluation of physiological consequences of cell engraftment, such as monitoring of the angiogenic response to therapy, which has recently been applied in the setting of myocardial infarction [28]. infarct border zones. d NOGA voltage mapping and linear local shortening (LLS) plots of the left ventricle revealed impaired electrical activity within region of myocardial infarction, which was used to guide transplantation of transfected and control cells that are identified as $\mathbf{e}$ dark red spots on a volume rendering of LLS. f Volume rendering of quadruple fusion of CTA, SPECT ${ }^{99 \mathrm{~m}}$ Tc-tetrofosmin, SPECT ${ }^{123}$ I, and NOGA demonstrated localization of intramyocardial cell injections to infarct border zones, with control cell injection sites not associated with focal uptake of ${ }^{123}$ I. Reprinted with permission of [22] (Color figure online)

Further development of hybrid imaging approaches such as PET-MR, which provide high resolution and low radiation characteristics, should continue to expand the application of nuclear imaging technology.

\section{CT Imaging}

CT imaging has been a primary non-invasive diagnostic technique since initial development and application in the 1960s and 1970s. Three-dimensional CT images are created using X-rays that are emitted from a source, transmitted 
through a patient, and detected by a detector array [29]. CT imaging possesses excellent penetration depth and spatial resolution, as well as high contrast for visualizing certain anatomical structure, particularly bone [30], making CT a useful imaging tool for evaluating structural and morphological changes in tissue-engineered constructs implanted in patients that have contraindications for MR. However, $\mathrm{X}$-ray sources emit ionizing radiation that can damage DNA, and iodinated contrast agents for CT imaging can be toxic for patients with impaired renal function. Although CT is useful for bone and vascular imaging, this technique is limited by suboptimal contrast within soft tissue. To date, CT is usually applied in the evaluation of regenerative medicine through pairing with targeted molecular imaging approaches such as SPECT and PET.

Although CT is not currently used as a stand-alone imaging modality for molecular imaging, targeted contrast agents are currently in development for clinical translation [29]. Standard CT contrast agents approved for clinical use are composed of iodinated small molecules or barium suspensions that possess a short half-life in blood. The characteristics of CT contrast agents make them ideally suited for cardiac and vascular imaging; however, recent research has been focused on the development of nanoparticles that possess longer blood pool circulation time, which may offer the ability to track transplanted cells in vivo and target-specific molecular processes. Nanoparticles containing various types of coating material, such as polymer, lipid, protein, and silica have been investigated pre-clinically to increase blood halflife and modulate solubility in blood and tissue [31-34] with coatings that can be specifically tailored to include different targeting moieties [29, 35, 36]. Additionally, nanoparticles can be formulated to contain materials capable of generating high levels of contrast that would otherwise not be attainable with small molecules such as iodine [37, 38]. CT technology for tracking of transplanted cells in vivo continues to experience challenges due to poor sensitivity of $\mathrm{CT}$ and complications associated with contrast loading of transplanted cells; however, pre-clinical efforts are continuing to evolve and show potential for clinical application [39-41].

Spectral CT is a new imaging approach that may assist in clinical translation of targeted CT imaging of regenerative medicine therapies through the differentiation of various contrast agents or materials simultaneously by recognition of the X-ray energy associated with each respective material. Multiple imaging channels can be set to match the K-edges of materials in targeted tissues of interest and generate color-coded images [42]. Spectral CT has been used to distinguish materials such as gold, iodine, bismuth, ytterbium, gadolinium, and calcified tissue [29]. Pre-clinical application has demonstrated the ability to simultaneous characterize vessel stenosis, calcification, and macrophage content within atherosclerotic plaque, suggesting that spectral CT may be useful for evaluating tissue-engineered vascular grafts and bioabsorbable stents [43]. Currently, CT imaging represents a complementary tool for molecular imaging approaches; however, as CT technologies continue to develop, new opportunities for targeted CT imaging of regenerative therapies may be recognized.

\section{MR Imaging}

MR has been a widely applied modality in the clinical setting due to numerous advantages, such as excellent penetration depth, high soft tissue contrast, and safety. MR uses strong magnetic fields (1.5-10 Tesla) that are capable of polarizing hydrogen nuclei within water molecules in a variety of tissues. Through the process of sending and receiving radio frequency pulse sequences, MR generates high-resolution images that are sensitive for evaluating anatomy, function, and pathology without the use of ionizing radiation [2].

In the evaluation of tissue-engineering applications, MR is an attractive imaging modality due to the ability to serially evaluate morphological changes in engineered constructs. To date, MR has been used in clinical trials to monitor stenosis and graft failure in tissue-engineered pulmonary valves [44] as well as neovascularization within bovine bone matrix implanted in a sinus lift procedure [45]. Further application of MR sequences, such as phase contrast imaging for the evaluation of luminal flow and wall shear stress characteristics, could lead to further progress in the evaluation of tissue-engineered heart valves and vascular grafts [46].

One unique characteristic of MR that continues to appeal to researchers involved in translational regenerative medicine is the ability to non-invasively track transplanted cells using superparamagnetic iron oxide nanoparticles (SPIONs). Evaluation of successful cell engraftment and migration can be detected through visualization of decreased signal intensity generated from SPION-labeled cells. The benefits associated with nanoparticle-based MR cell tracking include relatively easy cell labeling, high-resolution imaging of cell migration and homing of injected cells, and cell labeling persistence [12]. To date, SPIONs have been incorporated in a variety of regenerative clinical trials focused on various organ systems, such as the brain [10], spinal cord [11], and liver [7••, 47]. In cell therapy for patients with traumatic brain injury, SPION labeling techniques have been successful at tracking cell migration for 21 days and generating a detectable MR signal of cell engraftment for up to 7 weeks [10]. MR imaging has also been applied in patients with diabetes to track migration of SPION-labeled pancreatic islets transplanted into the liver and demonstrated a stable MR signal from labeled islets for 
up to 24 weeks, which was associated with considerable improvements in insulin dependence following transplant $[7 \bullet \bullet, 47]$. In addition to tracking of transplanted cells in brain and liver tissue, MR imaging has also exhibited the capacity to serially monitor SPION-labeled CD34+ cells in patients with spinal cord injury for 35 days following transplant [11] as well as evaluate cell migration of SPION-labeled dendritic cells transplanted into lymph nodes of melanoma patients [4]. Although SPION-based labeling strategies have proven to be an effective strategy for tracking transplanted cells in clinical trials, MR imaging of SPIONs is not without limitations. The prolonged presence of certain SPIONs may result in macrophages consuming SPIONs following the death of transplanted cells, which could lead to reduced reliability of long-term tracking of cell engraftment with MR [48]. Recently, Yilmaz et al. [49] exploited the role of macrophages in the inflammatory process using SPIONbased MR imaging of ferucarbotran as a tool for tracking inflammation and visualizing the peri-infarct zone in patients with acute myocardial infarction. Although no clinical trials to date have attempted to track labeled stem cells in the heart with MR, clinical translation of MR tools such as cardiac diffusion tensor imaging (DTI) tractography, which allows for visualization of myofiber alignment following cell transplantation in the heart, may offer novel methods for evaluating the effect of cell therapies following myocardial infarction [50].

In addition to nanoparticle-labeled cell imaging, MR has the potential for evaluating the functional outcomes associated with cell transplantation in various organs. Brain plasticity, cell differentiation, and re-myelination can be assessed using functional MR [51], MR spectroscopy [52, 53], and diffusion MR imaging [54]. Cardiac diffusion tensor imaging has demonstrated reproducibility for evaluating myocyte orientation in patients with hypertrophic cardiomyopathy [55]. Additionally, contrast-enhanced MR imaging with gadolinium has been used to identify a decrease in infarct size and an increase in tissue viability in the myocardium following stem cell therapy in heart failure [56••] and post-myocardial infarction patients [57, 58].

Although MR offers a high-resolution, high-contrast imaging modality for tracking of transplanted cells and evaluation of structure and function, SPECT and PET remain the primary imaging modalities for targeted molecular imaging due to the low sensitivity and lack of molecular probes available for MR. Ongoing developments in PET/MR systems should provide future opportunities for tracking transplanted cells and associated molecular events through improved co-localization of high-resolution MR imaging with high-sensitivity molecular imaging. In addition to the development of novel hybrid imaging systems incorporating MR, clinical trials are already utilizing MR as a tool for guiding targeted cell delivery [59-61]. Continued progress in the field of MR should lead to improved safety and quality control of cell transplantation for future regenerative trials.

\section{Ultrasound}

Ultrasound is a commonly used non-invasive imaging modality that is capable of generating real-time images of structure, function, and blood flow for various organ systems through the use of reflections and echoes of an oscillating sound wave on tissues [2]. Because of the relatively low cost and easy portability of ultrasound systems, clinicians are able to acquire high spatial and temporal resolution bedside images. Although ultrasound has been a widely accepted clinical imaging tool for many years, ultrasound-based techniques for performing targeted imaging have experienced slow progress. One molecular ultrasound strategy that has been developed that has potential for clinical regenerative medicine applications is contrast-enhanced imaging. Contrast agents that have been created for clinical ultrasound imaging primarily consist of gas-filled, lipid-shelled microbubbles. Due to the relatively large size of microbubbles (1-4 $\mu \mathrm{m}$ diameter), they are trapped within the intravascular space and are capable of imaging the microvasculature, thus restricting their use to disease processes that are associated with vascular endothelial cells, such as angiogenesis, inflammation, and thrombus formation [62]. Microbubbles have potential for targeted imaging via coating with polymers or proteins and can also serve as chemotherapeutic agents by being packaged with various pharmaceuticals [63]. Along with microbubbles, gas-filled (perfluorocarbon) nanoparticles have been developed for ultrasound, demonstrating favorable ultrasound-specific imaging characteristics and the ability to target cell-bound proteins [64]. Although ultrasound is capable of performing high-resolution imaging at high frequencies, the limited depth penetration of frequency waves has thus far prevented the clinical application of contrast-enhanced molecular ultrasound for in vivo tracking of transplanted cells [2]. However, ongoing research is focused on developing targeted contrast agents for clinical translation [65-67].

While molecular ultrasound has not proven to be an effective tool for tracking transplanted cells, ultrasound has demonstrated potential for evaluating bioabsorbable tissueengineered vascular grafts in clinical trials [68, 69]. Mechanical stability, graft patency, blood flow, wall degradation, and aneurysm risk are various parameters that have been evaluated with serial ultrasound in autologous [68] and allogenic [69] tissue-engineered vascular grafts. The recent development of intravascular ultrasound (IVUS), which is a miniaturized ultrasound transducer attached to the tip of an arterial catheter, now allows for intravascular imaging of vessel and lumen dimensions, as well as plaque evolution 
and morphology. Currently, IVUS is being applied in patients to evaluate serial changes in atherosclerotic development in bioresorbable scaffolds used for coronary revascularization [70] and has been incorporated in clinical trials to assist with the deployment of drug-eluting coronary stents [71•]. Additionally, application of other ultrasound technologies, such as intracardiac echocardiography (ICE), may improve the guidance of targeted cell delivery in the myocardium of patients following myocardial infarction in future clinical trials [72].

Ultrasound systems continue to evolve and be integrated with various imaging techniques such as near-infrared spectroscopy and photoacoustic imaging, which should ultimately enhance the future application of ultrasound in the evaluation of tissue-engineered vascular grafts and bioabsorbable and drug-eluting stents [63, 73]. Ongoing development of targeted contrast agents and nanoparticles for molecular ultrasound may facilitate the clinical translation of ultrasound as a tool for non-invasive assessment of regenerative medicine therapies.

\section{Optical Coherence Tomography}

In addition to IVUS, optical coherence tomography (OCT) is another imaging modality that is becoming increasingly used for intravascular imaging in the clinical setting. OCT generates images from the emission and reflection of near-infrared light and possesses the highest spatial resolution of all existing imaging modalities, with approximately tenfold greater resolution than ultrasound-based techniques $(10-15 \mu \mathrm{m}$ axial and 20-25 $\mu \mathrm{m}$ lateral). Although OCT possesses superior spatial resolution, this imaging modality is limited by poor penetration depth through blood and tissue (1-3 mm) [73]. Initial time domain OCT (TD-OCT) systems offered limited capability for intravascular imaging due to scatter and attenuation of OCT light by red blood cells. To avoid issues with image signal, early TD-OCT clinical imaging required a balloon occlusion of the vessel of interest combined with a transparent flushing medium for acquisition of intravascular images. Frequency-domain OCT and optical frequencydomain technology have since been developed, which now allows for focused, rapid imaging of long arterial segments without the need for balloon occlusions, thus eliminating the risk for myocardial ischemia or vessel wall damage [74-76]. Due to the greater spatial resolution of OCT when compared to IVUS, OCT may improve guidance and evaluation of vascular stent deployment. Specifically, the ability of OCT to identify under- or over-expansion of deployed stents, intrastent tissue protrusion, stent edge dissection, and intrastent thrombus may enhance the safety and guidance of interventional procedures in the future. To date, clinical trials have incorporated OCT to evaluate edge dissection following stent deployment [77] and compared OCT- to angiography-guided percutaneous coronary intervention [78•], demonstrating the safety and feasibility of using OCT imaging for procedures in interventional cardiology. Continued application of OCT will be required to fully elucidate if this technology is associated with improved clinical outcomes and is superior to IVUS for guidance and evaluation of stent and vascular graft implantation. Ongoing development of devices for regenerative medicine, such as bioabsorbable stents and tissue-engineered vascular grafts, should provide new opportunities for clinical application of intravascular imaging with OCT.

\section{Conclusions}

To date, clinical imaging modalities provide a variety of methods for evaluation of regenerative medicine therapies; however, no singular technique exists that offers a perfect all-in-one approach for tracking high-resolution anatomical and high-sensitivity molecular information following transplantation of tissue-engineered constructs or cell- and gene-based therapies. Limitations and questions still remain with regard to the safety and efficacy of delivery methods and optimization of imaging approaches before non-invasive serial tracking of regenerative therapies is optimized for clinical trials. Although many questions remain unanswered, non-invasive imaging should facilitate the future evaluation and clinical translation of novel regenerative medicine therapies.

Acknowledgments This work was supported in part by American Heart Association award 14CRP20480404 to Mitchel R. Stacy.

\section{Compliance with Ethics Guidelines}

Conflict of Interest Mitchel R. Stacy and Albert J. Sinusas have no conflicts of interest to declare.

Human and Animal Rights and Informed Consent This article does not contain any studies with human or animal subjects performed by any of the authors.

\section{References}

Papers of interest that have been recently published are highlighted as:

- Of importance

•• Of major importance

1. Polykandriotis E, Popescu LM, Horch RE (2010) Regenerative medicine: then and now-an update of recent history into future possibilities. J Cell Mol Med 14:2350-2358

2. Naumova AV, Modo M, Moore A et al (2014) Clinical imaging in regenerative medicine. Nat Biotechnol 32:804-818 
3. Stacy MR, Maxfield MW, Sinusas AJ (2012) Targeted molecular imaging of angiogenesis in PET and SPECT: a review. Yale $\mathrm{J}$ Biol Med 85:75-86

4. De Vries IJ, Lesterhuis WJ, Barentsz JO et al (2005) Magnetic resonance tracking of dendritic cells in melanoma patients for monitoring of cellular therapy. Nat Biotechnol 23:1407-1413

5. Kang WJ, Kang HJ, Kim HS et al (2006) Tissue distribution of 18F-FDG-labeled peripheral hematopoietic stem cells after intracoronary administration in patients with myocardial infarction. J Nucl Med 47:1295-1301

6. Schachinger V, Aicher A, Dobert N et al (2008) Pilot trial on determinants of progenitor cell recruitment to the infarcted human myocardium. Circulation 118:1425-1432

7. • Saudek F, Jirak D, Girman P et al (2010) Magnetic resonance imaging of pancreatic islets transplanted into the liver in humans. Transplantation 90:1602-1606. This study demonstrates the ability of MR to track long-term engraftment of SPION-labeled pancreatic islets transplanted in the liver of diabetic patients

8. Eich T, Eriksson O, Lundgren T, Transplantation NN for CI (2007) Visualization of early engraftment in clinical islet transplantation by positron-emission tomography. N Engl J Med 356:2754-2755

9. Eriksson O, Eich T, Sundin A et al (2009) Positron emission tomography in clinical islet transplantation. Am J Transpl 9:2816-2824

10. Zhu J, Zhou L, XingWu F (2006) Tracking neural stem cells in patients with brain trauma. N Engl J Med 355:2376-2378

11. Callera F, de Melo CM (2007) Magnetic resonance tracking of magnetically labeled autologous bone marrow CD34+ cells transplanted into the spinal cord via lumbar puncture technique in patients with chronic spinal cord injury: CD34+ cells' migration into the injured site. Stem Cells Dev 16:461-466

12. Nguyen PK, Riegler J, Wu JC (2014) Stem cell imaging: from bench to bedside. Cell Stem Cell 14:431-444

13. Kooreman NG, Ransohoff JD, Wu JC (2014) Tracking gene and cell fate for therapeutic gain. Nat Mater 13:106-109

14. Sinusas AJ, Thomas JD, Mills G (2011) The future of molecular imaging. JACC Cardiovasc Imaging 4:799-806. doi:10.1016/j. jemg.2011.05.003

15. Stacy MR, Naito Y, Maxfield MW et al (2014) Targeted imaging of matrix metalloproteinase activity in the evaluation of remodeling tissue-engineered vascular grafts implanted in a growing lamb model. J Thorac Cardiovasc Surg. 148(5):2227-2233. doi:10.1016/j.jtcvs.2014.05.037

16. Hofmann M, Wollert KC, Meyer GP et al (2005) Monitoring of bone marrow cell homing into the infarcted human myocardium. Circulation 111:2198-2202

17. Karpov RS, Popov SV, Markov VA et al (2005) Autologous mononuclear bone marrow cells during reparative regeneration after acute myocardial infarction. Bull Exp Biol Med 140:640-643

18. •• Vrtovec B, Poglajen G, Lezaic L et al (2013) Comparison of transendocardial and intracoronary CD34+ cell transplantation in patients with nonischemic dilated cardiomyopathy. Circulation 128:S42-S49. This clinical trial evaluated the retention of ${ }^{99 m}{ }^{T-}$ labeled CD34+ cells in the myocardium using two different modes of cell delivery

19. Yaghoubi SS, Jensen MC, Satyamurthy N et al (2009) Noninvasive detection of therapeutic cytolytic T cells with $18 \mathrm{~F}-\mathrm{FHBG}$ PET in a patient with glioma. Nat Clin Pr Oncol 6:53-58

20. Dohan O, De la Vieja A, Paroder V et al (2003) The sodium/ iodide symporter (NIS): characterization, regulation, and medical significance. Endocr Rev 24:48-77

21. •- Templin C, Zweigerdt R, Schwanke K et al (2012) Transplantation and tracking of human-induced pluripotent stem cells in a pig model of myocardial infarction: assessment of cell survival, engraftment, and distribution by hybrid single photon emission computed tomography/computed tomography of sodium iodide symporter transgene expression. Circulation 126:430-439. This pre-clinical study demonstrated successful non-invasive imaging of NIS-transfected cells for up to 15 weeks following intramyocardial transplantation in a porcine model of myocardial infarction

22. Fiechter M, Ghadri JR, Sidler M et al (2012) Cardiac quadruplefusion imaging: a brief report on a novel integrated multimodality approach for in vivo visualization of transplanted stem cells. Int $\mathbf{J}$ Cardiol 161:62-63

23. •- Rosado-de-Castro PH, Schmidt Fda R, Battistella V et al (2013) Biodistribution of bone marrow mononuclear cells after intra-arterial or intravenous transplantation in subacute stroke patients. Regen Med 8:145-155. This study demonstrates the initial feasibility of tracking cell delivery to the brain of patients following stroke

24. Battistella V, de Freitas GR, da Fonseca LM et al (2011) Safety of autologous bone marrow mononuclear cell transplantation in patients with nonacute ischemic stroke. Regen Med 6: $45-52$

25. Mendez I, Sanchez-Pernaute R, Cooper O et al (2005) Cell type analysis of functional fetal dopamine cell suspension transplants in the striatum and substantia nigra of patients with Parkinson's disease. Brain 128:1498-1510

26. Piccini P, Brooks DJ, Bjorklund A et al (1999) Dopamine release from nigral transplants visualized in vivo in a Parkinson's patient. Nat Neurosci 2:1137-1140

27. •• Kefalopoulou Z, Politis M, Piccini P et al (2014) Long-term clinical outcome in fetal cell transplantation for Parkinson disease: two case reports. JAMA Neurol 71:83-87. This study establishes the effectiveness of PET imaging of dopaminergic innervation in evaluating patients up to 18 years following cell transplantation in the brain

28. Mozid AM, Holstensson M, Choudhury T et al (2014) Clinical feasibility study to detect angiogenesis following bone marrow stem cell transplantation in chronic ischaemic heart failure. Nucl Med Commun 35:839-848

29. Cormode DP, Naha PC, Fayad ZA (2014) Nanoparticle contrast agents for computed tomography: a focus on micelles. Contrast Media Mol Imaging 9:37-52

30. Fleischmann D, Boas FE (2011) Computed tomography-old ideas and new technology. Eur Radiol 21:510-517

31. Moghimi SM, Hunter AC, Murray JC (2001) Long-circulating and target-specific nanoparticles: theory to practice. Pharmacol Rev 53:283-318

32. Torchilin VP, Frank-Kamenetsky MD, Wolf GL (1999) CT visualization of blood pool in rats by using long-circulating, iodine-containing micelles. Acad Radiol 6:61-65

33. Mukundan SJ, Ghaghada KB, Badea CT et al (2006) A liposomal nanoscale contrast agent for preclinical CT in mice. AJR Am J Roentgenol 186:300-307

34. Hill ML, Corbin IR, Levitin RB et al (2010) In vitro assessment of poly-iodinated triglyceride reconstituted low-density lipoprotein: initial steps toward CT molecular imaging. Acad Radiol 17: 1359-1365

35. Javier DJ, Nitin N, Levy M et al (2008) Aptamer-targeted gold nanoparticles as molecular-specific contrast agents for reflectance imaging. Bioconjug Chem 19:1309-1312

36. Pan D, Roessi E, Schlomka JP et al (2010) Computed tomography in color: nanoK-enhanced spectral CT molecular imaging. Angew Chem Int Ed Engl 49:9635-9639

37. Corot C, Robert P, Idee JM, Port M (2006) Recent advances in iron oxide nanocrystal technology for medical imaging. Adv Drug Deliv Rev 58:1471-1504

38. Cormode DP, Skajaa T, Fayad ZA, Mulder WJM (2009) Nanotechnology in medical imaging: probe design and applications. Arterioscler Thromb Vasc Biol 29:992-1000 
39. Criscione JM, Dobrucki LW, Zhuang ZW et al (2011) Development and application of a multimodal contrast agent for SPECT/CT hybrid imaging. Bioconjug Chem 22:1784-1792

40. Kedziorek DA, Solaiyappan M, Walczak P et al (2013) Using $\mathrm{C}$-arm X-ray imaging to guide local reporter probe delivery for tracking stem cell engraftment. Theranostics 3:916-926

41. Fu Y, Azene N, Ehtiati $\mathrm{T}$ et al (2014) Fused X-ray and MR imaging guidance of intrapericardial delivery of microencapsulated human mesenchymal stem cells in immunocompetent swine. Radiology 272:427-437

42. Schlomka JP, Roessl E, Dorscheid R et al (2008) Experimental feasibility of multi-energy photon-counting K-edge imaging in pre-clinical computed tomography. Phys Med Biol 53:4031-4047

43. Cormode DP, Roessl E, Thran A et al (2010) Atherosclerotic plaque composition: analysis with multicolor CT and targeted gold nanoparticles. Radiology 256:774-782

44. Voges I, Brasen JH, Entenmann A et al (2013) Adverse results of a decellularized tissue-engineered pulmonary valve in humans assessed with magnetic resonance imaging. Eur J Cardiothorac Surg 44:e272-e2279

45. Sauerbier S, Palmowski M, Vogeler M et al (2009) Onset and maintenance of angiogenesis in biomaterials: in vivo assessment by dynamic contrast-enhanced MRI. Tissue Eng Part C Methods $15: 455-462$

46. Von Knobelsdorff-Brenkenhoff F, Trauzeddel RF, Barker AJ et al (2014) Blood flow characteristics in the ascending aorta after aortic valve replacement-a pilot study using 4D-flow MRI. Int J Cardiol 170:426-433

47. Toso C, Vallee JP, Morel P et al (2008) Clinical magnetic resonance imaging of pancreatic islet grafts after iron nanoparticle labeling. Am J Transpl 8:701-706

48. Terrovitis J, Stuber M, Youssef A et al (2008) Magnetic resonance imaging overestimates ferumoxide-labeled stem cell survival after transplantation in the heart. Circulation 117: $1555-1562$

49. Yilmaz A, Rosch S, Klingel K et al (2013) Magnetic resonance imaging (MRI) of inflamed myocardium using iron oxide nanoparticles in patients with acute myocardial infarction-preliminary results. Int J Cardiol 163:175-182

50. Sosnovik EE, Mekkaoui C, Huang S et al (2014) Microstructural impact of ischemia and bone marrow-derived cell therapy revealed with diffusion tensor magnetic resonance imaging tractography of the heart in vivo. Circulation 129:1731-1741

51. Lazaridou A, Astrakas L, Mintzopoulos D et al (2013) fMRI as a molecular imaging procedure for the functional reorganization of motor systems in chronic stroke. Mol Med Rep 8:775-779

52. Ross BD, Hoang TQ, Bluml S et al (1999) In vivo magnetic resonance spectroscopy of human fetal neural transplants. NMR Biomed 12:221-236

53. Chung YL, El Akabawy G, So PW et al (2013) Profiling metabolite changes in the neuronal differentiation of human striatal neural stem cells using $1 \mathrm{H}$-magnetic resonance spectroscopy. NeuroReport 24:1035-1040

54. Gupta N, Henry RG, Strober J et al (2012) Neural stem cell engraftment and myelination in the human brain. Sci Transl Med 4:155ra137

55. McGill LA, Ismail TF, Nielles-Vallespin S et al (2012) Reproducibility of in vivo diffusion tensor cardiovascular magnetic resonance in hypertrophic cardiomyopathy. J Cardiovasc Magn Reson 14:86

56. • Chugh AR, Beache GM, Loughran JH et al (2012) Administration of cardiac stem cells in patients with ischemic cardiomyopathy: the SCIPIO trial: surgical aspects and interim analysis of myocardial function and viability by magnetic resonance. Circulation 126:S54-S64. This study demonstrates the usefulness of MR imaging for tracking serial changes in infarct size and cardiac function following cardiac stem cell therapy

57. Makkar RR, Smith RR, Cheng K et al (2012) Intracoronary cardiosphere-derived cells for heart regeneration after myocardial infarction (CADUCEUS): a prospective, randomised phase 1 trial. Lancet 379:895-904

58. Malliaras K, Makkar RR, Smith RR et al (2014) Intracoronary cardiosphere-derived cells after myocardial infarction: evidence of therapeutic regeneration in the final 1-year results of the CADUCEUS trial. J Am Coll Cardiol 63:110-122

59. Donovan T, Fryer TD, Pena A et al (2003) Stereotactic MR imaging for planning neural transplantation: a reliable technique at 3 Tesla? Br J Neurosurg 17:443-449

60. Kondziolka D, Steinberg GK, Cullen SB, McGrogan M (2004) Evaluation of surgical techniques for neuronal cell transplantation used in patients with stroke. Cell Transpl 13:749-754

61. Muir KW, Sinden J, Miljan E, Dunn L (2011) Intracranial delivery of stem cells. Transl Stroke Res 2:266-271

62. Deshpande N, Needles A, Willman JK (2010) Molecular ultrasound imaging: current status and future directions. Clin Radiol 65:567-581

63. O'Donnell M, McVeigh ER, Strauss HW et al (2010) Multimodality cardiovascular molecular imaging technology. J Nucl Med 51:38S-50S

64. Winter PM, Cai K, Caruthers SD et al (2007) Emerging nanomedicine opportunities with perfluorocarbon nanoparticles. Expert Rev Med Devices 4:137-145

65. Wei S, Fu N, Sun Y et al (2014) Targeted contrast-enhanced ultrasound imaging of angiogenesis in an orthotopic mouse tumor model of renal carcinoma. Ultrasound Med Biol 40:1250-1259

66. Pochon S, Tardy I, Bussat P et al (2010) BR55: a lipopeptidebased VEGFR2-targeted ultrasound contrast agent for molecular imaging of angiogenesis. Invest Radiol 45:89-95

67. Pysz MA, Foygel K, Rosenberg J et al (2010) Antiangiogenic cancer therapy: monitoring with molecular US and a clinically translatable contrast agent (BR55). Radiology 256:519-527

68. McAllister TN, Maruszewski M, Garrido SA et al (2009) Effectiveness of haemodialysis access with an autologous tissueengineered vascular graft: a multicentre cohort study. Lancet 373:1440-1446

69. Wystrychowski W, McAllister TN, Zagalski K et al (2014) First human use of an allogenic tissue-engineered vascular graft for hemodialysis access. J Vasc Surg 60(5):1353-1357. doi:10.1016/ j.jvs.2013.08.018

70. Lee IS, Bourantas CV, Muramatsu T et al (2013) Assessment of plaque evolution in coronary bifurcations located beyond everolimus eluting scaffolds: serial intravascular ultrasound virtual histology study. Cardiovasc Ultrasound 11:25

71. - Chieffo A, Latib A, Caussin C et al (2013) A prospective, randomized trial of intravascular-ultrasound guided compared to angiography guided stent implantation in complex coronary lesions: the AVIO trial. Am Heart J 165:65-72. This study evaluates the potential of IVUS for guiding the deployment of drug-eluting stents in patients with complex coronary lesions

72. Bartel T, Muller S, Biviano A, Hahn RT (2014) Why is intracardiac echocardiography helpful? Benefits, costs, and how to learn. Eur Heart J 35:69-76

73. Jegere S, Narbute I, Erglis A (2014) Use of intravascular imaging in managing coronary artery disease. World J Cardiol 6:393-404

74. Yun SH, Boudoux C, Tearney GJ, Bouma BE (2003) High-speed wavelength-swept semiconductor laser with a polygon-scannerbased wavelength filter. Opt Lett 28:1981-1983

75. De Boer JF, Cense B, Park BH et al (2003) Improved signal-tonoise ratio in spectral-domain compared with time-domain optical coherence tomography. Opt Lett 28:2067-2069 
76. Tearney GJ, Waxman S, Shishkov M et al (2008) Three-dimensional coronary artery microscopy by intracoronary optical frequency domain imaging. JACC Cardiovasc Imaging 1:752-761

77. Gonzalo N, Serruys PW, Okamura T et al (2009) Optical coherence tomography assessment of the acute effects of stent implantation on the vessel wall: a systematic quantitative approach. Heart 95:1913-1919

78. • Prati F, Di Vito L, Biondi-Zoccai G et al (2012) Angiography alone versus angiography plus optical coherence tomography to guide decision-making during percutaneous coronary intervention: the Centro per la Lotta contro l'Infarto-Optimisation of Percutaneous Coronary Intervention (CLI-OPCI) study. Eurointervention 8:823-829. This observational clinical trial demonstrates that OCT may improve guidance of percutaneous coronary interventions 